\title{
Statins as adjunct therapy in COPD: how do we cope after STATCOPE?
}

\author{
Robert P Young, ${ }^{1}$ Raewyn J Hopkins, ${ }^{1}$ Alvar Agusti ${ }^{2}$
}

\section{INTRODUCTION}

Statins are inhibitors of 3-hydroxy-3methylglutaryl coenzyme A reductase that are widely used to reduce cardiovascular (CVS) disease risk yet have pleiotropic effects in other organs, including the lungs. ${ }^{1} 2$ The results of the Simvastatin for the Prevention of Exacerbations in Moderate-to-Severe COPD (STATCOPE) study, that investigated their potential effects on exacerbations of COPD, have been recently published in the New England Journal of Medicine. ${ }^{3}$ In this Hot off the breath editorial, we review the rationale for the study, discuss its design, main results, strengths and limitations, and speculate on the future of statins in COPD.

\section{RATIONALE: HINTS FROM OBSERVATIONAL STUDIES}

Statins reduce the recruitment of neutrophils and macrophages into the lung, alter bronchial remodelling, prevent emphysema and reduce systemic inflammation. ${ }^{1} \quad 2 \quad 4$ These biological effects appear to have clinical relevance as observational studies in COPD patients report reduced all-cause mortality, ${ }^{5-8}$ reduced mortality from acute exacerbations, ${ }^{8-10}$ reduced frequency of COPD exacerbations, ${ }^{8}{ }^{11-13}$ and reduced decline of lung function, ${ }^{14}$ for those taking statins, as compared to not taking them.

In one small randomised controlled trial (RCT), statin use in COPD patients was associated with a clinically significant increase in exercise tolerance, although this benefit was limited to those in whom baseline C-reactive protein (CRP) was elevated (ie, those with evidence of systemic inflammation). ${ }^{15}$ To date, STATCOPE is the only RCT that has assessed the effect of adjunct statin treatment prospectively on acute exacerbations in COPD in a large cohort of patients. ${ }^{3}$

${ }^{1}$ School of Biological Science and the Faculty of Medical and Health Sciences, University of Auckland, Auckland, New Zealand; ${ }^{2}$ Thorax Institute, Hospital Clinic, IDIBAPS, University of Barcelona, and CIBERES, Madrid, Spain

Correspondence to Dr RP Young, University of Auckland, P.O. Box 26161 Epsom, Auckland 1344, New Zealand; roberty@adhb.govt.nz.

\section{STATCOPE FINDINGS: THE BASIS OF DISCORDANT RESULTS}

STATCOPE was a large, randomised, multicentre, double-blind, placebo-controlled trial of simvastatin $(40 \mathrm{mg} /$ day $)$ in the prevention of COPD exacerbations in patients with moderate-to-severe COPD $(\mathrm{N}=885) .^{3}$ Importantly, patients with diabetes or CVS disease (figure 1), those already on statins, or those that required statins based on accepted CVS risk criteria, were excluded. The primary outcome of the study was negative, as simvastatin treatment for between 12 and 36 months was no more effective than placebo in reducing the frequency or severity of COPD exacerbations. ${ }^{3}$ Moreover, lung function and quality of life were not different between groups. ${ }^{3}$

So why did observational studies observe a $30-50 \%$ reduction in COPD exacerbations with statin therapy, ${ }^{8}$ 11-13 whereas STATCOPE found none? These discordant results raise issues about the strengths and weaknesses of observational data (reflecting 'real-world' statin use in an uncontrolled setting) over RCT data (reflecting statin use in a highly selected COPD subgroup in a controlled and highly monitored setting). These are discussed in detail below.

\section{OBSERVATIONAL STUDIES: NO EVIDENCE FOR A 'HEALTHY USER EFFECT'}

The first possibility is that statin therapy does not really reduce the frequency of COPD exacerbations, and that the observational study results were confounded by variables not adequately adjusted or matched for. However, a careful review of these observational studies show that lung function, cumulative smoking exposure, Body Mass Index, COPD-related medication use, vaccination use or socioeconomic status were similar in COPD patients prescribed statins versus non-users. $^{5-13}$ Hence, a 'healthy user effect' seems unlikely to account for the observed effects. By contrast, statin users invariably (albeit not surprisingly) had significantly greater diabetes $(26 \% \text { vs } 11 \%)^{12}$ and CVS disease prevalence, including arterial hypertension $(52 \%$ vs $34 \%),{ }^{12}$ heart failure $(12 \%$ vs $8 \%),{ }^{11}$ coronary artery disease (51\% vs $24 \%) .{ }^{10}$ That statin use in these observational studies was consistently associated with better outcomes in COPD patients with more comorbid diseases, ${ }^{5-13}$ effectively excludes 'confounding by drug indication' where outcomes would be worse (not better) in patients with comorbid CVS-related disease.

\section{OBSERVATIONAL STUDIES AND THE EVIDENCE FOR AN 'UNHEALTHY NON-USER EFFECT'}

A more plausible explanation is that observational studies include a large percentage of COPD patients with co-existing CVS diseases who are 'non-users', but would benefit from statin therapy (figure 1). These patients are likely to do very poorly due to one or a combination of undertreated pulmonary inflammation, unrecognised systemic inflammation, or subclinical CVS disease. ${ }^{16-18}$ These comorbid phenotypes of COPD are strongly associated with an increased risk of hospitalisation with 'acute exacerbations' and greater mortality. ${ }^{16-18}$ Hence, it is possible that many COPD patients who have not been prescribed statins in observational studies do badly from undertreatment, a hypothesis suggested by the STATCOPE investigators to explain the discordant results. ${ }^{3}$ Data from the observational studies, which include COPD patients outside hospital outpatient clinics, indicate that about $30-40 \%$ of all patients with COPD are prescribed statin therapy. ${ }^{2-13}$ If a significant proportion of the remaining $60-70 \%$ of COPD patients not prescribed statins would benefit from statin therapy, then the non-use of statins in the observational studies would be associated with poor outcomes and attributed to an 'unhealthy non-user effect' rather than a 'healthy user effect' (figure 1). Of note, of those COPD patients not taking statins in the observational studies, 11-25\% had diabetes, 34-50\% had hypertension, $13-24 \%$ had coronary heart disease (CHD) and a surprising $41 \%$ had 'cardiovascular disease'. ${ }^{10-12}$ This suggests that as much as one half of COPD patients not taking statins, for clinically apparent CVS disease, probably should be based on their cardiovascular risk profiles alone. This estimate concurs with that calculated by STATCOPE investigators (see Protocol). ${ }^{3}$ If this were true, then the 1.5-2.0-fold greater mortality, which has been consistently reported in statin non-users in observational studies, ${ }^{5-7}$ would be entirely expected.

\section{PRIMARY PREVENTION IN COPD: SHOULD WE ADD UNDERTREATMENT TO UNDERDIAGNOSIS?}

A plausible explanation for the STATCOPE findings is that statin therapy 
Figure 1 Proposed schema of the COPD patients participating in observational studies and STATCOPE, stratified according to the presence of comorbid CVS diseases* , background statin use and presence of systemic inflammation (SI). The diameter of each circle is roughly proportional to the percentage of patients in each group (shown below each one). The overlapping circles on the left (yellow=overt CVS disease and red=subclinical, or high risk of, CVS disease) indicate that some patients with CVS diseases are not treated with statins, whereas others with high CVS risk are for primary prevention. By contrast, the right hand circle has no overlap since no patient in this group is on statins given their low CVS risk. ${ }^{*}$ CVS diseases include: IHD, ischaemic heart disease; PVD, peripheral vascular disease; CVD, cerebrovascular disease; $\mathrm{CHF}$, congestive heart failure,

\# unhealthy non-user effect.

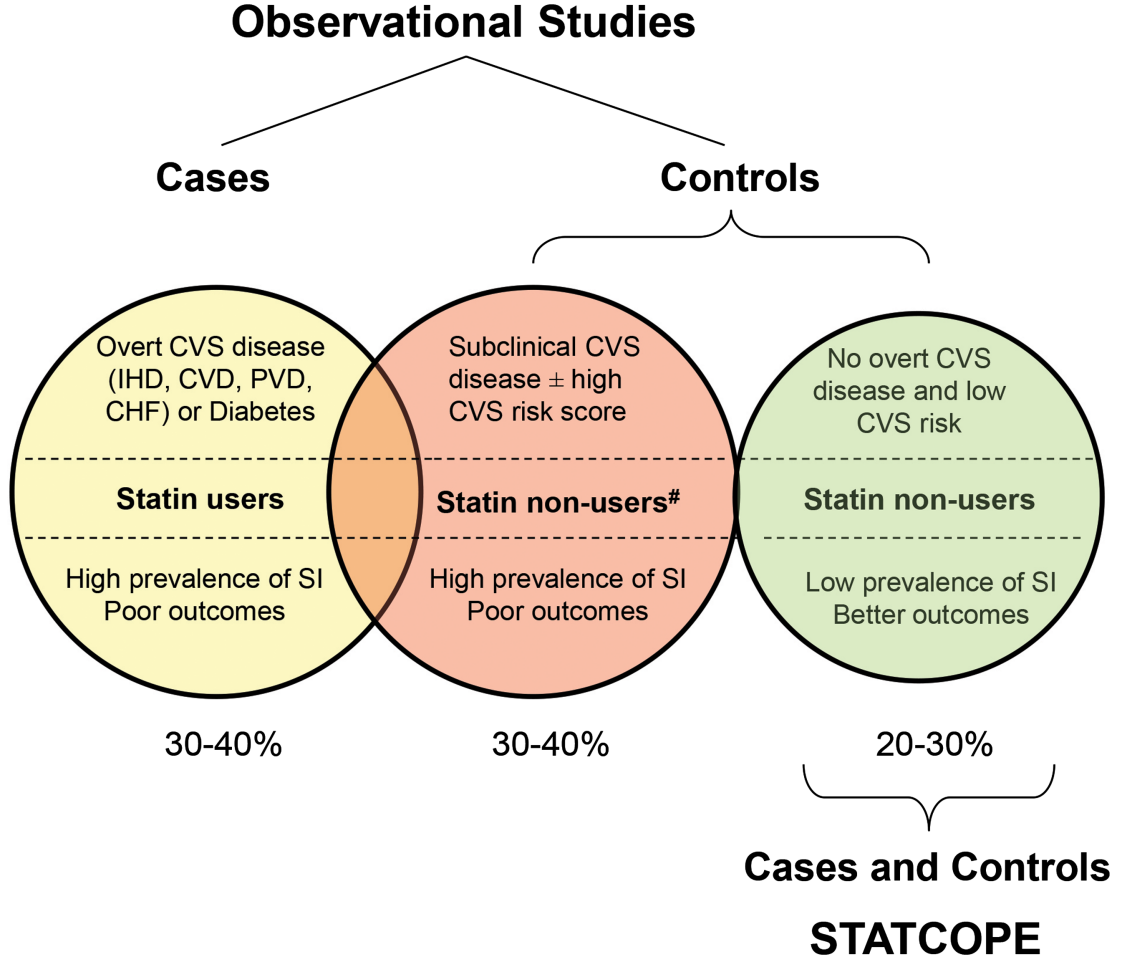

has no effect on reducing 'acute exacerbations' in COPD patients where coexisting clinical and subclinical CVS disease has been all but excluded. This is important, as recent studies suggest that the role of cardiovascular comorbidity underlying 'acute exacerbations' of COPD, in particular heart failure, may be much greater than previously recognised. ${ }^{19-21}$ These studies suggest between $30 \%$ and $50 \%$ of admissions attributed to an acute exacerbation may be due, in part, to 'cardiac dysfunction'. ${ }^{20}$ Systemic inflammation has also been linked in prospective studies to congestive heart failure in the absence of clinically obvious coronary artery disease. $^{21}$ Given the findings of the STATCOPE study, it might be reasonable to conclude that statin therapy is primarily indicated in those patients with COPD where their cardiovascular risk justifies its use according to established Framingham-based guidelines (this might include as much as $60-70 \%$ of all COPD patients (see later)). However, such a conclusion makes two potentially incorrect assumptions. First, that COPD is not itself an independent predictor of CVS disease (eg, like diabetes). Yet, there is evidence indicating that reduced $\mathrm{FEV}_{1}$ independently conferred just as great a risk of $\mathrm{CHD}$ as increased serum cholesterol, ${ }^{22}$ where statins are routinely recommended in the absence of overt coronary disease. Second, that statins do not have a beneficial effect on mortality independent of that attributed to the treatment of CVS diseases in patients with COPD. The observational studies reported so far have consistently found that all-cause mortality in unselected COPD patients is improved by $30-50 \%$ with statin therapy compared to those not taking statins. ${ }^{5-7}$ While this might be attributed solely to reduced CVS deaths, ${ }^{1}$ in three observational studies, a reduction in 'respiratory-related' deaths was also found, ${ }^{579}$ although the accuracy of death certification remains a limitation of these findings. The observation that statin therapy reduces mortality, and specifically respiratory-related mortality, in COPD patients requires confirmation in a RCT.

\section{SELECTION OF COPD PATIENTS IN STATCOPE: IS STATCOPE} GENERALISABLE TO THE WIDER COPD POPULATION?

STATCOPE excluded patients on statins as well as those who should be on statins based on their 'cardiovascular risk profiles' ${ }^{3}$ This may have removed the very COPD patients who might benefit most from statin therapy. Such an exclusionary approach might therefore leave a largely lower risk and potentially 'statin-unresponsive' group recruited into the STATCOPE study where any benefit might be limited (figure 1). Several features of STATCOPE support such a possibility. It is notable that reduction of low-density lipoprotein in STATCOPE, with $40 \mathrm{mg}$ of simvastatin, was only $23 \%$, and less than the expected $36-40 \%$ reduction normally seen with this dose. ${ }^{23}$ Mortality in the placebo arm of STATCOPE over 3 years was $6 \%$, only half that reported in TORCH over a similar time period. ${ }^{24}$ Additionally, the frequency of comorbid cardiovascularrelated diseases in the STATCOPE participants has not been reported but is presumably very low or non-existent. This brings into question the generalisability of the STATCOPE findings to COPD populations in general, where overt or subclinical cardiovascular disease may collectively affect as much as $75 \%$ of all patients. ${ }^{3-13}$ ${ }^{25}$ Given participants in STATCOPE were prescreened through medical record data, it is impossible to estimate what proportion of COPD patients were excluded due to their cardiovascular profile alone. Based on observational studies of comorbid disease in COPD, ${ }^{5-13} 26$ it may have been as much as $30-40 \%$ which, in addition to the $30-40 \%$ taking statins (as estimated by STATCOPE investigators), ${ }^{3}$ leaves only $20-30 \%$ 'lower risk' COPD patients eligible for STATCOPE (figure 1).

\section{STATCOPE AND EFFECT OF SIMVASTATIN: WHAT'S HAPPENING TO SYSTEMIC INFLAMMATION?}

A further possibility underlying the STATCOPE findings is that any benefit from statin therapy in reducing COPD exacerbations may be primarily confined to those patients with coexisting systemic inflammation. ${ }^{3}$ Recent results suggest between $40 \%$ and $70 \%$ of patients with 
stable COPD have elevation of at least one marker of systemic inflammation. ${ }^{27} 28$ Further, two published RCTs on exercise tolerance in COPD, ${ }^{15} 28$ and one observational study on mortality, ${ }^{7}$ suggest that the statin-derived benefits in COPD are almost exclusively observed in those with systemic inflammation arbitrarily defined as a CRP greater than $3 \mathrm{mg} / \mathrm{L}^{7} 1528 \mathrm{~A}$ secondary (still unpublished but much awaited) analysis of STATCOPE will look at the effects of simvastatin on patients with systemic inflammation (see online supplementary materials from Criner et al). ${ }^{3}$ However, if the exclusion criteria of STATCOPE effectively removed those with elevated cardiovascular risk (figure 1), it is possible (or even likely) that many patients with systemic inflammation had been excluded too. If so, underpowering from small sample size may become an issue, particularly since studies in the cardiovascular literature have shown that the effect of $40 \mathrm{mg}$ of simvastatin on reducing systemic inflammation is related to the baseline CRP value: null in those with $\mathrm{CRP}<1 \mathrm{mg} / \mathrm{L}$ (in whom CRP actually goes up), 16\% in those with CRP values $1-3 \mathrm{mg} / \mathrm{L}$, and $32 \%$ in those with CRP $>3 \mathrm{mg} / \mathrm{L}^{29}$

\section{STATCOPE AND THE QUESTION OF STUDY DESIGN}

Another possible explanation for the discordant findings between the observational studies and STATCOPE is that other relevant differences between the COPD populations under consideration may have diminished any beneficial effect of statins in the latter (figure 1). First, in STATCOPE, nearly $73 \%$ of COPD patients were taking inhaled corticosteroids (ICS) compared to a mean of 7\% (range 4-77\%) in the observational studies. ${ }^{3}$ 10-12 Furthermore, in STATCOPE, COPD patients were contacted during the study on a monthly basis with telephone calls or clinic visits, where drug compliance was closely monitored. ${ }^{3}$ Given that ICS may reduce pulmonary and systemic inflamma$\operatorname{tion}^{30}$ if taken by the majority of STATCOPE participants, it is possible that little additional benefit for COPD exacerbations was gained by adding simvastatin in this closely monitored cohort. Second, according to the STATCOPE protocol, it appears that any patients who met exclusion criteria based on the risk-based eligibility for statin therapy during follow-up, may be prescribed simvastatin and continue in the study on an intention-to-treat basis. ${ }^{3}$ What proportion of patients originally randomised to placebo, received statins during follow-up as per the safety-based treatment cross-over, is not stated in the STATCOPE publication. If it was large, a dilutional effect on outcome may have resulted. Finally, in most other aspects, the STATCOPE COPD patients were representative of moderate to severe COPD, although supplementary oxygen use (significantly greater at $>40 \%$ ) and duration of statin therapy (significantly shorter with $56 \%<2$ years) were also substantially different to that reported in the observational studies.

\section{LIFE AFTER STATCOPE WITH STATINS 'DOWN BUT NOT OUT': WHERE TO FROM HERE?}

So what should readers of Thorax make of the findings? There is no argument that statin therapy is indicated in patients with COPD who have clinically overt CHD (secondary prevention) or those at high risk of CVS diseases (primary prevention). Based on the results of the JUPITER trial $^{31}$ the latter might include those clinically stable COPD patients with elevated systemic inflammation (CRP $>3 \mathrm{mg} / \mathrm{L})$. At this time, there is no RCT evidence to support statin use in reducing acute exacerbations of COPD. The secondary analysis of STATCOPE based on systemic inflammatory biomarkers may help clarify the usefulness of simvastatin in this setting, although exclusion of those patients at high risk of CVS disease and systemic inflammation may dilute a potentially beneficial effect. Given that limited RCT data found improvement with exercise tolerance and quality of life measures in those with the systemic inflammatory phenotype, ${ }^{15} 28$ and that observational data suggests that significant reductions in mortality remains a potential benefit, ${ }^{5-10}$ more RCTs are urgently needed to better examine the potential benefits of statins as adjunct therapy in COPD.

Contributors All authors contributed to the writing of this article and approve this final version.

Competing interests None.

Provenance and peer review Commissioned; internally peer reviewed.

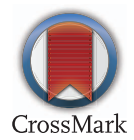

To cite Young RP, Hopkins RJ, Agusti A. Thorax 2014;69:891-894

Received 12 June 2014

Accepted 19 June 2014

Published Online First 11 July 2014

Thorax 2014;69:891-894.

doi:10.1136/thoraxinl-2014-205814

\section{REFERENCES}

1 Zhou Q, Liao JK. Pleiotropic effects of statins: basic research and clinical perspectives. Circ $J$ 2010;74:818-26.

2 Young RP, Hopkins RJ, Eaton TE. Pharmacological actions of statins: potential utility in COPD. Eur Respir Rev 2009:18:222-32.

3 Criner GJ, Connett JE, Aaron SD, et al. Simvastatin for the prevention of exacerbations in moderate-to-severe COPD. N Eng J Med 2014:370:2201-10.

4 Wright JL, Zhou S, Preobrazhenska O, et al. Statin reverses smoke-induced pulmonary hypertension and prevents emphysema but not airway remodelling. Am J Respir Crit Care Med 2011;183:50-8.

5 Frost FJ, Petersen H, Tollestrup K, et al. Influenza and COPD mortality protection as pleiotropic, dose-dependent effects of statins. Chest 2007;131:1006-12.

6 Lawes CMM, Thornley $S$, Young $R$, et al. Statin use in COPD patients is associated with a reduction in mortality: a national cohort study. Prim Care Respir 2012:21:35-40.

7 Lahousse L, Loth DW, Joos GF, et al. Statins, systemic inflammation and risk of death in COPD: The Rotterdam study. PulmPharmacol Ther 2013:26:212-17.

8 Mancini GBJ, Etminan M, Zhang B, et al. Reduction of morbidity and mortality by statins, angiotensin-converting enzyme inhibitors, and angiotensin receptor blockers in patients with chronic obstructive pulmonary disease. J Am Coll Cardiol 2006:47:2554-60.

9 Young RP, Hopkins RJ, Chan W, et al. Effect of statin therapy on mortality in COPD: analysis of cause-specific deaths in a National cohort study. Am J Respir Crit Care Med 2013;187:A6017.

10 Bartziokas K, Papaioannou Al, Minas M, et al. Statins and outcome after hospitalization for COPD exacerbation: a prospective study. Pulm Pharmacol Ther 2011;24:625-31.

11 Wang M-T, Lo Y-W, Tsai C-L, et al. Statin use and risk of COPD exacerbations requiring hospitalization. Am J Med 2013;126:598-606.

12 Huang $C-C$, Chan W-L, Chen Y-C, et al. Statin use and hospitalization in patients with chronic obstructive pulmonary disease: a nationwide population-based cohort study in Taiwan. Clin Ther 2011:33:1365-70.

13 Blamoun Al, Batty GN, DeBari VA, et al. Statins may reduce episodes of exacerbation and the requirement for intubation in patients with COPD: evidence from a retrospective cohort study. Int I Clin Pract 2008:62:1373-8.

14 Alexeeff SE, Litonjua AA, Sparrow D, et al. Statin use reduces decline in lung function: VA Normative Aging Study. Am J Respir Crit Care Med 2007;176: 742-7.

15 Lee T-M, Lin M-S, Chang N-C. Usefulness of C-reactive protein and interleukin- 6 as predictors of outcomes in patients with chronic obstructive pulmonary disease receiving pravastatin. $\mathrm{Am} J$ Cardiol 2008;101:530-5.

16 Dahl M, Vestbo J, Lange $\mathrm{P}$, et al. C-reactive protein as a predictor of prognosis in chronic obstructive pulmonary disease. Am I Respir Crit Care Med 2007:175:250-5.

17 Fabbri LM, Beghe B, Agusti A. Cardiovascular mechanisms of death in severe COPD exacerbation: time to think and act beyond guidelines. Thorax 2011:66:745-7.

18 Maclay JD, Mc Nee W. Cardiovascular disease in COPD: mechanisms. Chest 2013;143: 798-807.

19 Sharif R, Parekh TM, Pierson KS, et al. Predictors of early re-admission among patients aged 40-64 years hospitalised for chronic obstructive pulmonary disease. Annals ATS 2014:11:685-94. 
20 Nishimura K, Nishimura T, Onishi K, et al. Changes in plasma levels in B-type natriuretic peptide with acute exacerbations of chronic obstructive pulmonary disease. Int I COPD 2014:9:155-62.

21 Vasan RS, Sullivan LM, Roubenoff $R$, et al. Inflammatory markers and risk of heart failure in elderly subjects without prior myocardial infarction: The Framingham Heart Study. Circulation 2003:107:1486-91.

22 Hole DJ, Watt GCM, Davey-Smith G, et al. Impaired lung function and mortality risk in men and women: findings from the Renfrew and Paisley prospective population study. BMJ 1996:313:711-15.

$23 \mathrm{http}: / / w w w . c l e v e l a n d c l i n i c m e d e d . c o m / m e d i c a l p u b s /$ disaesemanagement/cardiology. Cardiovascular Disease Prevention (accessed 6 Jun 2014).
24 Calverley PMA, Anderson JA, Celli B, et al. Salmeterol and fluticasone proprionate and survival in chronic obstructive pulmonary disease. $N$ Eng J Med 2007;356:775-89.

25 Soyeth V, Brekke PH, Smith P, et al. Statin use is associated with reduced mortality in COPD. Eur Respir J 2007;29:279-83.

26 Vanfleteran LEGW, Spruit MA, Groenen M, et al. Clusters of comorbidities based on validated objective measurements and systemic inflammation in patients with chronic obstructive pulmonary disease. Am J Respir Crit Care Med 2013;187:728-35.

27 Agusti A, Edwards LD, Rennard SI, et al. Persistent systemic inflammation is associated with poor clinical outcomes in COPD: a novel phenotype. PloS ONE 2012;7:e37483.
28 Young RP, Hopkins RJ. Update on the potential role of statins in chronic obstructive pulmonary disease and its co-morbidities. Expert Rev Respir Med 2013;7:533-44.

29 Pearson T, Ballantyne C, Sisk C, et al. Comparison of effects of Ezitimibe/simvastatin versus simvastatin versus atorvastatin in reducing $\mathrm{C}$-reactive protein and low-density lipoprotein cholesterol levels. Am J Cardiol 2007;99:1706-13.

30 Sin DD, Lacy P, York E, et al. Effects of fluticasone on systemic markers of inflammation in chronic obstructive pulmonary disease. Am I Respir Crit Care Med 2004;170:760-5.

31 Ridker PM, Danieson E, Fonseca FAH, et al. Rosuvastatin to prevent vascular events in men and women with elevated C-reactive protein. $N$ Eng $J$ Med 2008;359:2195-207. 